\title{
KNOWLEDGE AND RISK PERCEPTION OF NIGERIANS TOWARDS THE CORONAVIRUS DISEASE (COVID-19)
}

Bolaji Felicia Udomah ${ }^{1}$, Uriel Oludare Ashaolu ${ }^{2}$ Charles Oluwatemitope Olomofe ${ }^{3}$, Olufunke Folasade Dada ${ }^{4}$, Victor Kehinde Soyemi ${ }^{5}$, Yetunde Bolatito Aremu-Kasumu ${ }^{6}$, Chikezie John Ochieze ${ }^{7}$, Ayodele Olusola Adeyemi ${ }^{8}$, Adeyinka Olabisi Owolabi ${ }^{9}$, Martin Chukwudum Igbokwe ${ }^{10}$, Emmanuel Eziashi Ajumuka $^{11}$, Kehinde Williams Ologunde ${ }^{12}$, Gbenga Omotade Popoola $^{13}$ Olumuyiwa Elijah Ariyo ${ }^{14}$, Olaniyi Bamidele Fayemi ${ }^{15}$

${ }^{1}$ University of Osun Teaching Hospital, Osogbo, Osun State, Nigeria

${ }^{2}$ Obafemi Awolowo University Teaching Hospital Complex, Ile-Ife, Osun State, Nigeria

${ }^{3}$ Abt Associates (USAID SHOPS Plus TB Project), Nigeria

${ }^{4}$ Obafemi Awolowolo University Teaching Hospital Complex, Ile-Ife, Osun State, Nigeria

${ }^{5}$ Medical Affairs, Pharma R\&D, GlaxoSmithKline Pharmaceutical, Nigeria

${ }^{6}$ Federal Medical Center, Gusau, Zamfara State, Nigeria

${ }^{7}$ Detar Healthcare Systems, Victoria, Texas, United States

${ }^{8}$ Bafrow Medical Center, The Republic of the Gambia

${ }^{9}$ William Harvey Hospital, Willesborough, Ashford, Kent, United Kingdom

${ }^{10}$ Zenith Medical and Kidney Center, Kudu, Abuja, Nigeria

${ }^{11}$ Ahmadu Bello University Teaching Hospital, Zaria, Kaduna State, Nigeria

${ }^{12}$ Federal Medical Center, Gusau, Zamfara State, Nigeria 
medRxiv preprint doi: https://doi.org/10.1101/2021.07.30.21261351; this version posted August 1, 2021. The copyright holder for this preprint (which was not certified by peer review) is the author/funder, who has granted medRxiv a license to display the preprint in perpetuity. It is made available under a CC-BY-ND 4.0 International license .

${ }^{13}$ Department of Psychiatry, Federal Teaching Hospital, Ido-Ekiti, Ekiti State Nigeria

${ }^{14}$ Department of Medicine, Infectious Diseases and Tropical Medicine Unit, Federal

Teaching Hospital, Ido-Ekiti, Ekiti State, Nigeria

${ }^{15}$ Federal Teaching Hospital, Ido-Ekiti, Ekiti State, Nigeria

Corresponding author:Charles Oluwatemitope Olomofe, email-charlesolomofe@gmail.com

\begin{abstract}
ABSTARCT
Background: The Coronavirus Disease 2019 (COVID-19) is far from over, although appreciable progress has been made to limit the devastating effects of the pandemic across the globe. Adequate knowledge and risk perception is a critical assessment that is required to ensure proper preventive measures. This study assessed these among Nigerians.

Methods: The study was a cross-sectional assessment of 776 consenting Nigerian adults that were distributed across the 6 geo-political zones and the Federal Capital Territory. Online pretested, semi-structured questionnaire were used to obtain the socio-demographic data and assessed the knowledge and risk perception of the participants to COVID-19. The knowledge of COVID-19 was assessed based on the number of accurate responses given in comparison to average scores. Chi-square analysis was computed to analysis the association between sociodemographic characteristics and knowledge of COVID-19 and risk perception. Data analysis was done using SPSS version 21, the level of significance was set at value $p<0.05$ at $95 \%$ confidence interval.
\end{abstract}

Results: Majority of the participants were male $451(58.1 \%)$, there was a good knowledge of COVID-19 among $90.3 \%$ of respondents with $57 \%$ having positive risk perception. There was a statistically significant relationship between good knowledge and positive risk perception of COVID-19 ( $\mathrm{p}$ 0.001). Annual income $(\mathrm{p}=0.012)$ and the perception that "vaccines are good" significantly predict positive risk perception of COVID-19 among the respondents.

Conclusion: A good knowledge of COVID-19 and vaccination against the virus were the two most important factors that determined risk perception among the population. This may be because of the widespread advocacy, and it portends a good omen at combating COVID-19 menace.

Key words: COVID-19, Risk perception, Nigerians 
medRxiv preprint doi: https://doi.org/10.1101/2021.07.30.21261351; this version posted August 1, 2021. The copyright holder for this preprint (which was not certified by peer review) is the author/funder, who has granted medRxiv a license to display the preprint in perpetuity.

It is made available under a CC-BY-ND 4.0 International license .

\section{BACKGROUND}

The novel coronavirus (Severe Acute Respiratory Syndrome Coronavirus 2, SARS-CoV-2) was first reported in Wuhan city in China, and as of December 2020, 191 countries of the world have been affected by it. ${ }^{1}$ As of 16th January 2021, there were over 90 million confirmed cases worldwide with more than 2 million mortality while Nigeria had corresponding figures of 105 , 478, and 1,405 respectively. ${ }^{2,3}$ Coronaviruses are a large family of viruses that are known to cause illnesses ranging from the common cold to more severe diseases such as Middle East Respiratory Syndrome (MERS) and Severe Acute Respiratory Syndrome (SARS-CoV2). ${ }^{4}$ The clinical symptoms of Coronavirus Disease 2019 (COVID-19) include fever (which is the most common), cough, fatigue, malaise, and shortness of breath, ${ }^{5}$ and these symptoms are more severe amongst the elderly and those with underlying chronic medical conditions such as diabetes, chronic heart, and chronic lung diseases. 5,6

Many countries, including Nigeria experienced a second wave of the viral infection. The total number of COVID-19 infected persons is almost reaching 100,000. ${ }^{7}$ This becomes complicated because of new variants of the virus has also been discovered in Nigeria, which was first isolated in the United Kingdom (UK), said to be about $56 \%$ more transmissible, with a large number of mutation and virulence. ${ }^{8}$ Since the beginning of December there has been an astronomical increase in the number of new cases and deaths recorded on daily basis. ${ }^{9}$ Experts have linked this with non-adherence to the infection control recommendations. ${ }^{10}$ These recommendations are focused on disease prevention and control measures to minimise the spread as well as the burden on the healthcare system. ${ }^{11}$ These measures include frequent hand hygiene using soap and water or an alcohol-based sanitizer, keeping a physical distance of about 6 feet from others, quarantine for those who are exposed, use of face masks, and avoiding the touch of the face with unwashed 
medRxiv preprint doi: https://doi.org/10.1101/2021.07.30.21261351; this version posted August 1, 2021. The copyright holder for this preprint (which was not certified by peer review) is the author/funder, who has granted medRxiv a license to display the preprint in perpetuity.

It is made available under a CC-BY-ND 4.0 International license .

hands. ${ }^{12}$ However, many Nigerians still do not adhere to these preventive measures. Good knowledge, and risk perception which refers to the way the diseases is understood/interpreted, ${ }^{13}$ is important in pre-empting the way a person would react to an occurrence of the infection in their own lives as well as the lives of others. An understanding of the perception of people regarding COVID-19 is crucial because perception can act as a trigger for precautionary action, engagement in preventive health behaviour and reporting of suspected cases of COVID-19. ${ }^{14}$ Knowledge and perception of risk is influenced by both individual and societal factors which are based on experiences, beliefs, attitudes, social, cultural and institutional processes. ${ }^{13}$ Risk perception is also largely mediated by the information and knowledge that an individual has. Bhagavathula et $a{ }^{15}$ in their study assessed the awareness of COVID-19 among health care workers reported that knowledge of transmission (60\%) and disease onset (64\%) was poor among the health care workers.

The Nigerian government, non-governmental organizations (NGOs), and civil societies are using various avenues to bridge the knowledge gap by educating and enlightening the populace on COVID-19 and how to prevent its spread. One of such measures is the collaboration by the Nigerian Red Cross Society with tricycle riders in Maiduguri where they are providing stickers for them to use on their tricycles across the city. ${ }^{16}$ Also, several media strategies such as campaigns on social media platforms, press releases, radio jingles and drama skits in local dialects are being used. ${ }^{17}$ A survey conducted among Egyptians shows many had good knowledge of the disease but are not willing to practice the preventive measures. ${ }^{18}$ Anecdotal evidence suggests poor knowledge and poor perception of risk of the disease amongst the Nigerian population could be responsible for poor adherence to these preventive measures. ${ }^{19}$ Yet there is dearth of studies evaluating the knowledge and perception of the people about the on- 
medRxiv preprint doi: https://doi.org/10.1101/2021.07.30.21261351; this version posted August 1, 2021. The copyright holder for this preprint (which was not certified by peer review) is the author/funder, who has granted medRxiv a license to display the preprint in perpetuity.

It is made available under a CC-BY-ND 4.0 International license .

going COVID 19 pandemic among Nigerians. The elites in governance, policymakers and opinion leaders have been reported to have questioned the need for social distancing, while others have cited political, economic, and financial gains as the drive(s) for the fight against the infection. ${ }^{20}$ Similarly, a cross-section of people on the streets also gives overwhelming evidence that Nigerians are largely ignoring COVID-19 safety protocols. ${ }^{21}$ Therefore, there is a need to assess the level of knowledge of Nigerians about COVID-19 and their perceived risk of contracting the disease. The outcome of this research may reveal if their knowledge and risk perception of contracting COVID-19 is adequate or not and inform necessary action to improve the knowledge and risk perception targeted at proper adherence to preventive measures.

\section{METHODS}

This was a cross sectional study carried out in Nigeria, the most populated country in Africa with an estimated population of about 200 million and a total land area of 910,770 Km2 (351,650 sq. miles). ${ }^{22}$ Nigeria has six geopolitical zones (Southwest, Southeast, Southsouth, Northwest, Northeast, Northcentral) with 36 states. Ethical approval was gotten from the health research ethics committee of the Federal Medical Centre Gusau, Zamfara State.

This was an on-line study, conducted using a pre-tested, semi-structured questionnaire and included Nigerian adults above the age of 18 years who consented to participate in the study. The minimum sample size was determined to be 409 at confidence level of $95 \%$ and based on proportion of people with good knowledge of $39 \%$ in a previous study ${ }^{23}$ and a $5 \%$ margin of error.

The questionnaire was adapted from several published literature ${ }^{23-25}$ and covered three sections A-C that is, Socio-demographic characteristics of respondents, respondent's knowledge of 
medRxiv preprint doi: https://doi.org/10.1101/2021.07.30.21261351; this version posted August 1, 2021. The copyright holder for this preprint (which was not certified by peer review) is the author/funder, who has granted medRxiv a license to display the preprint in perpetuity.

It is made available under a CC-BY-ND 4.0 International license.

COVID 19, respondents risk perception of COVID 19. Pre-test of the questionnaire was done on $10 \%$ of the respondents each at six different states from the six geopolitical zones and were not included in the study. The pretested questionnaires with participants' information sheet were circulated extensively online.

Data was analyzed using SPSS version 21. The knowledge of COVID 19 among respondents was scored based on the number of accurate responses given. The number of correct responses were compared with the average score. Participants whose score equalled or was above the average were categorized as having good knowledge while those who scored below the average were categorized as having poor knowledge. Chi square analysis was computed to test for association between sociodemographic characteristics and knowledge of COVID 19 among respondents. The level of significance was predetermined at a p-value of less than 0.05 at $95 \%$ confidence level.

\section{RESULTS}

Table 1: Socio-demographic characteristics of Respondents

\begin{tabular}{|l|c|c|}
\hline Variables & Frequency $(\boldsymbol{n}=\mathbf{7 7 6})$ & Percent (\%) \\
\hline Age group (years) & & 12.0 \\
$18-25$ & 93 & 31.1 \\
$26-35$ & 241 & 43.9 \\
$36-45$ & 341 & 9.4 \\
$46-55$ & 73 & 3.6 \\
$>55$ & 28 & 58.1 \\
Gender & & 40.9 \\
Male & 451 & 1.0 \\
Female & 317 & 27.6 \\
Prefer not to say & 8 & 63.0 \\
Marital Status & 214 & 1.9 \\
Single & 489 & 0.9 \\
Married & 15 & 5.8 \\
Separated/Divorced & 7 & 0.8 \\
Widowed/ Widower & 45 & \\
In a relationship & 6 & \\
Undisclosed & & \\
\end{tabular}




\begin{tabular}{|c|c|c|}
\hline \multicolumn{3}{|l|}{ Religion } \\
\hline None & 9 & 1.2 \\
\hline Christianity & 616 & 79.4 \\
\hline Islam & 147 & 18.9 \\
\hline Others & 4 & 0.5 \\
\hline \multicolumn{3}{|l|}{ Level of Education } \\
\hline Secondary school or less & 36 & 4.6 \\
\hline Tertiary & 413 & 53.2 \\
\hline Postgraduate & 327 & 42.1 \\
\hline \multicolumn{3}{|l|}{ Occupation } \\
\hline Unemployed & 60 & 7.7 \\
\hline Trader & 22 & 2.8 \\
\hline Teacher & 67 & 8.6 \\
\hline Artisan & 17 & 2.2 \\
\hline Healthcare worker & 194 & 25.0 \\
\hline Information technology & 43 & 5.5 \\
\hline Financial services & 45 & 5.8 \\
\hline Security agent & 12 & 1.5 \\
\hline Agricultural services & 19 & 2.4 \\
\hline Others & 297 & 38.3 \\
\hline \multicolumn{3}{|c|}{ Estimated Income of Caregiver } \\
\hline \multicolumn{3}{|c|}{ Per Annum (Naira) } \\
\hline$<500,000$ & 136 & 17.5 \\
\hline 500,000 - 1 million & 120 & 15.5 \\
\hline $1-2$ million & 117 & 15.1 \\
\hline $2-3$ million & 54 & 7.0 \\
\hline $3-4$ million & 37 & 4.8 \\
\hline$>4$ million & 105 & 13.5 \\
\hline I prefer not to say & 207 & 26.7 \\
\hline \multicolumn{3}{|l|}{ State Origin (Zone) } \\
\hline North-Central & 90 & 11.6 \\
\hline North-East & 46 & 5.9 \\
\hline North-West & 49 & 6.3 \\
\hline South-East & 119 & 15.3 \\
\hline South-South & 94 & 12.1 \\
\hline South-West & 368 & 47.4 \\
\hline Prefer not to say & 10 & 1.3 \\
\hline \multicolumn{3}{|l|}{ Place of residence (Zone) } \\
\hline North-Central & 174 & 22.4 \\
\hline North-East & 24 & 3.1 \\
\hline North-West & 103 & 13.3 \\
\hline South-East & 32 & 4.1 \\
\hline South-South & 40 & 5.2 \\
\hline South-West & 357 & 46.0 \\
\hline Prefer not to say & 46 & 5.9 \\
\hline
\end{tabular}


medRxiv preprint doi: https://doi.org/10.1101/2021.07.30.21261351; this version posted August 1, 2021. The copyright holder for this preprint (which was not certified by peer review) is the author/funder, who has granted medRxiv a license to display the preprint in perpetuity. It is made available under a CC-BY-ND 4.0 International license .

\begin{tabular}{|l|c|c|}
$1-4$ & 375 & 48.3 \\
$5-8$ & 357 & 46.0 \\
$>8$ & 44 & 5.7 \\
\hline
\end{tabular}

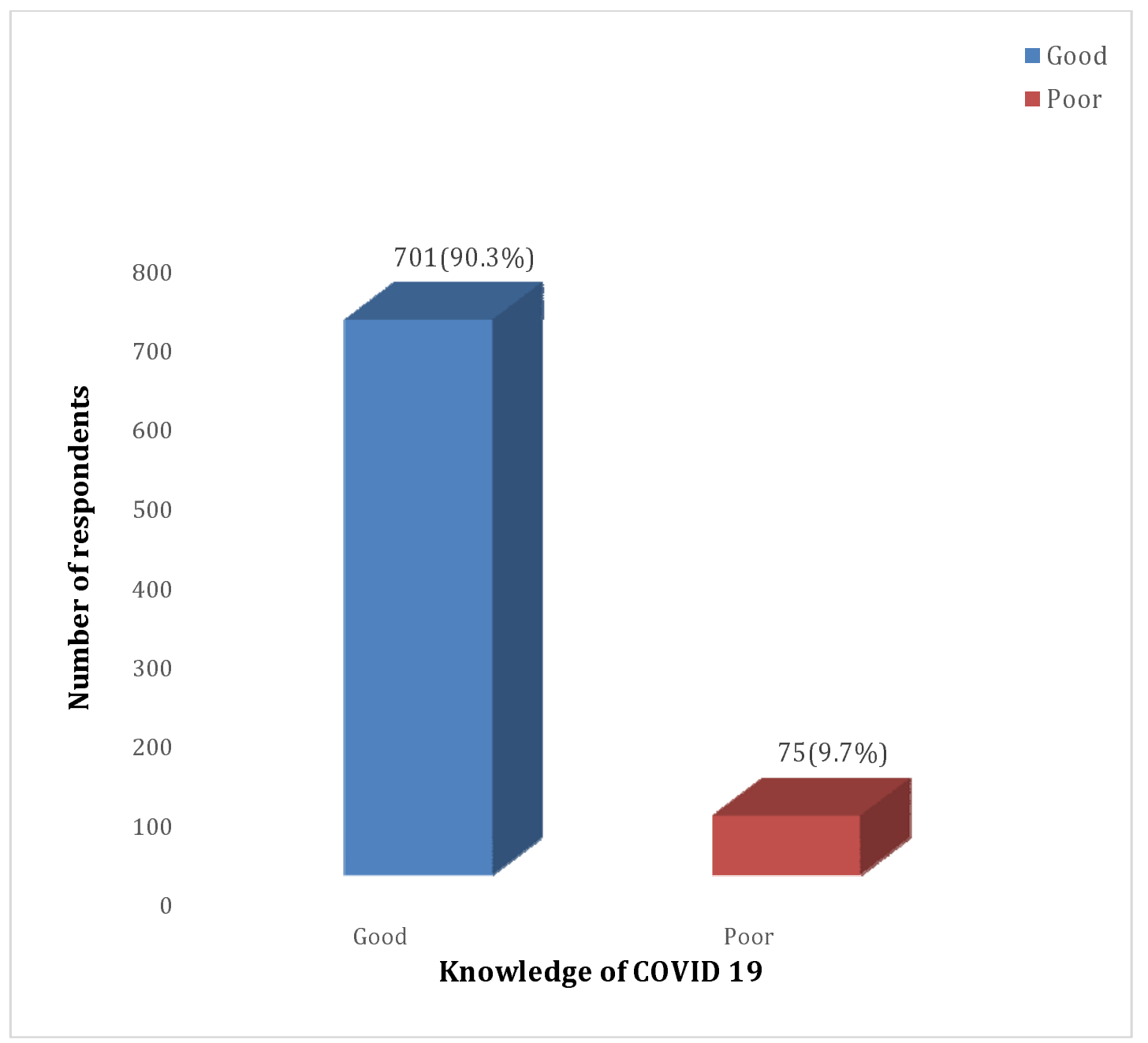

Fig 1: Overall knowledge of COVID-19 among respondents 
medRxiv preprint doi: https://doi.org/10.1101/2021.07.30.21261351; this version posted August 1, 2021. The copyright holder for this preprint (which was not certified by peer review) is the author/funder, who has granted medRxiv a license to display the preprint in perpetuity.

It is made available under a CC-BY-ND 4.0 International license .

Table 2: Respondents' source of awareness of COVID 19

\begin{tabular}{|l|c|c|}
\hline Source of awareness of COVID-19 & Frequency $(\boldsymbol{n}=$ & Percent (\%) \\
\hline Radio/television/newspaper/social media & $\mathbf{7 7 6})$ & 25.5 \\
Family/friends/neighbors & 198 & 0.6 \\
Healthcare workers/hospital/pharmacy & 5 & 1.5 \\
All the above & 12 & 71.8 \\
Others (Mosque, Church etc.) & 557 & 0.5 \\
\hline
\end{tabular}

Table 3: Risk Perception on COVID 19 among Respondents

\begin{tabular}{|c|c|c|c|c|c|c|}
\hline Risk Perception & $\begin{array}{c}\text { SA } \\
\text { n }(\%)\end{array}$ & $\begin{array}{c}\mathbf{A} \\
\mathbf{n}(\%)\end{array}$ & $\begin{array}{c}\mathbf{N} \\
\mathbf{n}(\%)\end{array}$ & $\begin{array}{l}\text { DA } \\
\text { n }(\%)\end{array}$ & $\begin{array}{l}\text { SD } \\
\text { n }(\%)\end{array}$ & $\begin{array}{l}\text { DK } \\
\text { n }(\%)\end{array}$ \\
\hline COVID-19 is a global disease & 711(91.6) & $50(6.4)$ & $7(0.9)$ & $2(0.3)$ & $2(0.3)$ & $4(0.5)$ \\
\hline $\begin{array}{l}\text { The disease can affect both adults } \\
\text { and children }\end{array}$ & $662(85.3)$ & $98(12.6)$ & $7(0.9)$ & $1(0.1)$ & $1(0.1)$ & $7(0.9)$ \\
\hline $\begin{array}{l}\text { I am doing enough to protect myself } \\
\text { and family }\end{array}$ & $480(61.9)$ & $250(32.2)$ & $31(4.0)$ & $5(0.6)$ & $1(0.1)$ & $9(1.2)$ \\
\hline $\begin{array}{l}\text { Government is doing enough to } \\
\text { protect myself and my family }\end{array}$ & $89(11.5)$ & 201(25.9) & $236(30.4)$ & $144(18.6)$ & $90(11.6)$ & $16(2.1)$ \\
\hline $\begin{array}{l}\text { Our healthcare system can handle } \\
\text { the disease }\end{array}$ & $71(9.1)$ & $150(19.3)$ & $246(31.7)$ & $186(24.0)$ & $103(13.3)$ & $20(2.6)$ \\
\hline $\begin{array}{l}\text { The disease is not a threat to human } \\
\text { race }\end{array}$ & $60(7.7)$ & $150(19.3)$ & $182(23.5)$ & $186(24.0)$ & $164(21.1)$ & $34(4.4)$ \\
\hline People can die from the disease & $624(80.4)$ & $112(14.4)$ & $16(2.1)$ & $6(0.8)$ & $6(0.8)$ & $12(1.5)$ \\
\hline $\begin{array}{l}\text { People can recover after having the } \\
\text { disease }\end{array}$ & $544(70.1)$ & $194(25.0)$ & $23(3.0)$ & $2(0.3)$ & $4(0.5)$ & $9(1.2)$ \\
\hline $\begin{array}{l}\text { It is only old people that die from } \\
\text { the disease }\end{array}$ & $22(2.8)$ & $93(12.0)$ & $182(23.5)$ & $251(32.3)$ & $174(22.4)$ & $54(7.0)$ \\
\hline $\begin{array}{l}\text { Even if am ill now I would not go to } \\
\text { the hospital because of the risk of } \\
\text { contracting the COVID infection }\end{array}$ & $98(12.6)$ & $181(23.3)$ & $230(29.6)$ & $145(18.7)$ & $90(11.6)$ & $32(4.1)$ \\
\hline
\end{tabular}

A = Agree, SA = Strongly Agree, N = Neutral, D = Disagree, SD = Strongly Disagree DK = Don't Know 
medRxiv preprint doi: https://doi.org/10.1101/2021.07.30.21261351; this version posted August 1, 2021. The copyright holder for this preprint (which was not certified by peer review) is the author/funder, who has granted medRxiv a license to display the preprint in perpetuity. It is made available under a CC-BY-ND 4.0 International license .

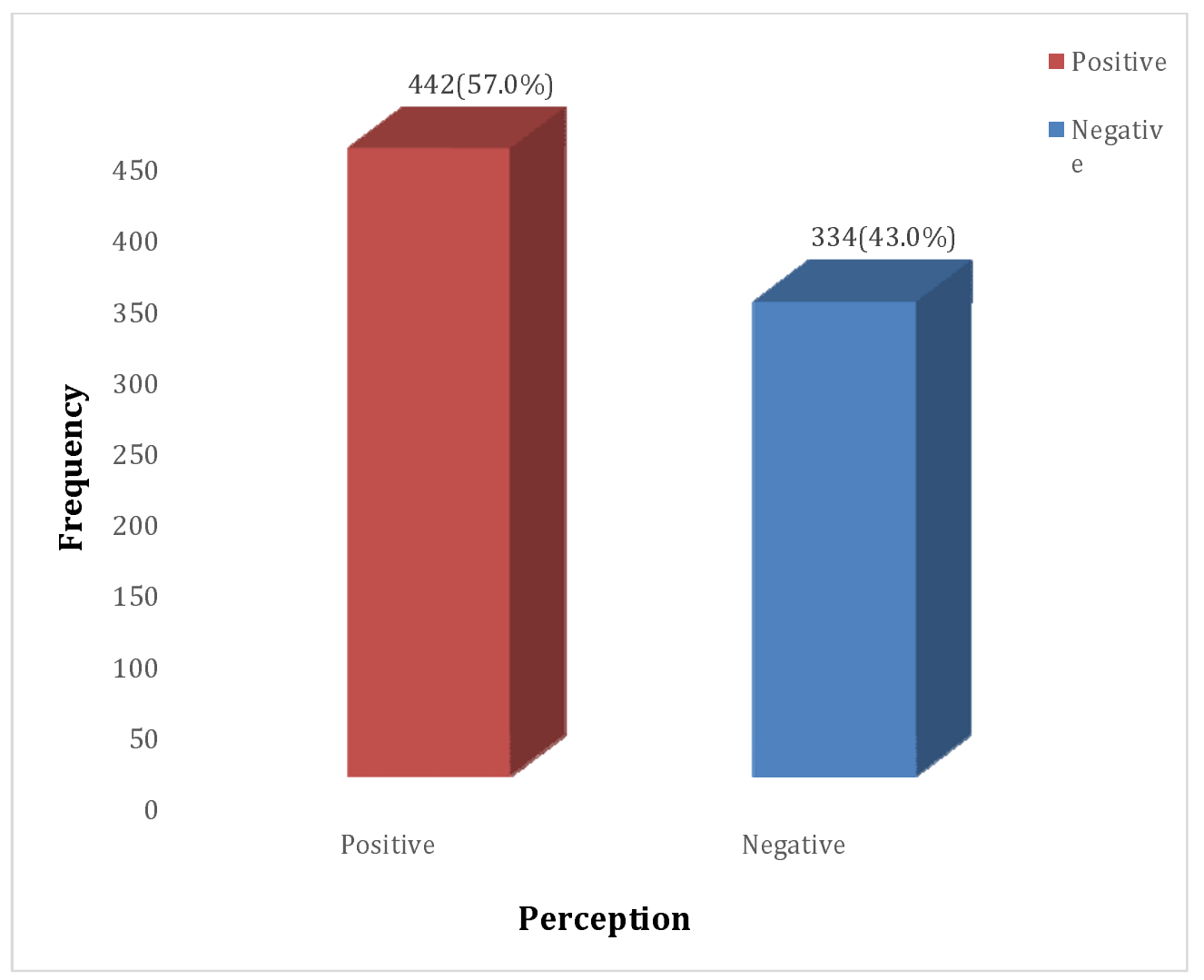

Fig 2: Overall risk perception of COVID-19 among respondents 
medRxiv preprint doi: https://doi.org/10.1101/2021.07.30.21261351; this version posted August 1, 2021. The copyright holder for this preprint (which was not certified by peer review) is the author/funder, who has granted medRxiv a license to display the preprint in perpetuity.

It is made available under a CC-BY-ND 4.0 International license .

Table 4a: Association between Sociodemographic Characteristics and Knowledge of COVID 19

\begin{tabular}{|c|c|c|c|c|c|}
\hline \multirow{2}{*}{ Socio-Demographic Characteristics } & \multicolumn{2}{|c|}{ Knowledge } & \multirow[b]{2}{*}{$\begin{array}{c}\text { Total } \\
\text { N }(\%) \\
\end{array}$} & \multirow[b]{2}{*}{$\chi^{2}$} & \multirow[b]{2}{*}{$p$-value } \\
\hline & $\begin{array}{l}\text { Good } \\
\text { n (\%) }\end{array}$ & $\begin{array}{l}\text { Poor } \\
\text { n }(\%)\end{array}$ & & & \\
\hline \multicolumn{6}{|l|}{ Age (years) } \\
\hline $18-25$ & $87(93.5)$ & $6(6.5)$ & 93 & 12.674 & 0.013* \\
\hline $26-35$ & $219(90.9)$ & $22(9.1)$ & 242 & & \\
\hline $36-45$ & $309(90.6)$ & $32(9.4)$ & 341 & & \\
\hline $46-55$ & $66(90.4)$ & $7(9.6)$ & 73 & & \\
\hline$>55$ & $20(71.4)$ & $8(28.6)$ & 28 & & \\
\hline \multicolumn{6}{|l|}{ Gender } \\
\hline Male & $411(91.1)$ & $40(8.9)$ & 451 & 0.514 & 0.473 \\
\hline Female & $284(89.6)$ & $33(10.4)$ & 317 & & \\
\hline \multicolumn{6}{|l|}{ Level education } \\
\hline Secondary school or less & $32(88.9)$ & $4(11.1)$ & 36 & 2.640 & 0.267 \\
\hline Tertiary & $367(88.9)$ & $46(11.1)$ & 413 & & \\
\hline Postgraduate & $302(92.4)$ & $25(7.6)$ & 327 & & \\
\hline \multicolumn{6}{|l|}{ Marital status } \\
\hline Single & $189(88.3)$ & $25(11.7)$ & 214 & $11.241^{\mathrm{F}}$ & $0.017 *$ \\
\hline Married & $446(91.2)$ & $43(8.8)$ & 489 & & \\
\hline Separated/ Divorced & $13(86.7)$ & $2(13.3)$ & 15 & & \\
\hline Widowed/ Widower & $4(57.1)$ & $3(42.9)$ & 7 & & \\
\hline In a relationship & $44(97.8)$ & $1(2.2)$ & 45 & & \\
\hline \multicolumn{6}{|l|}{ Number person in household } \\
\hline $1-4$ & $335(89.3)$ & $40(10.7)$ & 375 & 1.227 & 0.541 \\
\hline $5-8$ & $327(91.6)$ & $30(8.4)$ & 357 & & \\
\hline$>8$ & $39(88.6)$ & $5(11.4)$ & 44 & & \\
\hline \multicolumn{6}{|l|}{ State origin zone } \\
\hline North-Central & $78(86.7)$ & $12(13.3)$ & 90 & 10.440 & 0.064 \\
\hline North-East & $40(87.0)$ & $6(13.0)$ & 46 & & \\
\hline North-West & $43(87.8)$ & $6(12.2)$ & 49 & & \\
\hline South-East & $108(90.8)$ & $11(9.2)$ & 119 & & \\
\hline South-South & $80(85.1)$ & $14(14.9)$ & 94 & & \\
\hline South-West & $345(93.8)$ & $23(6.3)$ & 368 & & \\
\hline \multicolumn{6}{|l|}{ Place of residence } \\
\hline North-Central & $151(86.8)$ & $23(13.2)$ & 174 & $10.015^{\mathrm{F}}$ & 0.061 \\
\hline North-East & $21(87.5)$ & $3(12.5)$ & 24 & & \\
\hline North-West & $95(92.2)$ & $8(7.8)$ & 103 & & \\
\hline South-East & $30(93.8)$ & $2(6.3)$ & 32 & & \\
\hline South-South & $33(82.5)$ & $7(17.5)$ & 40 & & \\
\hline South-West & $333(93.3)$ & $24(6.7)$ & 357 & & \\
\hline \multicolumn{6}{|l|}{ Religion } \\
\hline None & $8(88.9)$ & $1(11.1)$ & 9 & 1.777 & 0.606 \\
\hline Christianity & $560(90.9)$ & $56(9.1)$ & 616 & & \\
\hline Islam & $129(87.8)$ & $18(12.2)$ & 147 & & \\
\hline Others & $4(100.0)$ & $0(0.0)$ & 4 & & \\
\hline
\end{tabular}


medRxiv preprint doi: https://doi.org/10.1101/2021.07.30.21261351; this version posted August 1, 2021. The copyright holder for this preprint (which was not certified by peer review) is the author/funder, who has granted medRxiv a license to display the preprint in perpetuity.

It is made available under a CC-BY-ND 4.0 International license.

Table 4b: Association between Sociodemographic Characteristics and Knowledge of COVID 19

\begin{tabular}{|c|c|c|c|c|c|}
\hline \multirow[b]{2}{*}{ Variable } & \multicolumn{3}{|c|}{ Knowledge } & \multirow[b]{2}{*}{$\chi^{2}$} & \multirow[b]{2}{*}{$p$-value } \\
\hline & $\begin{array}{l}\text { Good } \\
\text { n }(\%)\end{array}$ & $\begin{array}{l}\text { Poor } \\
\text { n }(\%)\end{array}$ & $\begin{array}{c}\text { Total } \\
\text { N }(\%)\end{array}$ & & \\
\hline \multicolumn{6}{|l|}{ Occupation } \\
\hline Unemployed & $53(88.3)$ & $7(11.7)$ & 60 & 19.348 & $\mathbf{0 . 0 2 2} *$ \\
\hline Trader & $22(100.0)$ & $0(0.0)$ & 22 & & \\
\hline Teacher & $53(79.1)$ & $14(20.9)$ & 67 & & \\
\hline Artisan & $16(94.1)$ & $1(5.9)$ & 17 & & \\
\hline Healthcare worker & $181(93.3)$ & $13(6.7)$ & 194 & & \\
\hline Information technology & $42(97.7)$ & $1(2.3)$ & 43 & & \\
\hline Financial services & $42(93.3)$ & $3(6.7)$ & 45 & & \\
\hline Security agent & $10(83.3)$ & $2(16.7)$ & 12 & & \\
\hline Agricultural services & $16(84.2)$ & $3(15.8)$ & 19 & & \\
\hline Others & $266(89.6)$ & $31(10.4)$ & 297 & & \\
\hline \multicolumn{6}{|l|}{ Average income } \\
\hline$<500,000$ & $122(89.7)$ & $14(10.3)$ & 136 & 6.434 & 0.266 \\
\hline $500,000-1$ million & $107(89.2)$ & $13(10.8)$ & 120 & & \\
\hline $1-2$ million & $108(92.3)$ & $9(7.7)$ & 117 & & \\
\hline $2-3$ million & $53(89.1)$ & $1(1.9)$ & 54 & & \\
\hline $3-4$ million & $36(97.3)$ & $1(2.7)$ & 37 & & \\
\hline$>4$ million & $97(92.4)$ & $8(7.6)$ & 105 & & \\
\hline
\end{tabular}

$\chi^{2}$ : Chi square test; F: Fisher's exact test; *: $p$ value $<0.05$ 
medRxiv preprint doi: https://doi.org/10.1101/2021.07.30.21261351; this version posted August 1, 2021. The copyright holder for this preprint (which was not certified by peer review) is the author/funder, who has granted medRxiv a license to display the preprint in perpetuity.

It is made available under a CC-BY-ND 4.0 International license .

Table 5: Association between knowledge of COVID-19 and risk perception of COVID-19

\begin{tabular}{lccccc}
\hline & \multicolumn{2}{c}{ Risk Perception of COVID 19} & & \\
Positive & Negative & Total & $\chi^{2}$ & p-value \\
Variable & $\mathbf{n}(\%)$ & $\mathbf{n}(\%)$ & $\mathbf{N}(\%)$ & & \\
\hline Knowledge of COVID-19 & & & & & \\
Good & $418(59.6)$ & $283(40.4)$ & 701 & 21.096 & $<\mathbf{0 . 0 0 1 *}$ \\
Poor & $24(32.0)$ & $51(68.0)$ & 75 & & \\
\hline
\end{tabular}

$\chi^{2}$ : Chi Square test; *: p value $<0.05$

TABLE 6: Predictors of positive risk perception of COVID-19

\begin{tabular}{|c|c|c|c|}
\hline Variable & B & p value & OR \\
\hline \multicolumn{4}{|l|}{ Age (years) } \\
\hline $18-25$ & 0.078 & 0.913 & $1.081(0.267-4.380)$ \\
\hline $26-35$ & 0.719 & 0.215 & $2.052(0.658-6.399)$ \\
\hline $36-45$ & 0.504 & 0.362 & $1.655(0.560-4.893)$ \\
\hline $46-55$ & 0.343 & 0.573 & $1.409(0.427-4.645)$ \\
\hline$>55^{\mathrm{REF}}$ & & & 1 \\
\hline \multicolumn{4}{|l|}{ Level education } \\
\hline Secondary school or less ${ }^{\text {REF }}$ & & & 1 \\
\hline Tertiary & 0.061 & 0.931 & $1.063(0.269-4.204)$ \\
\hline Postgraduate & -0.078 & 0.914 & $0.925(0.223-3.833)$ \\
\hline \multicolumn{4}{|l|}{ Marital status } \\
\hline Single ${ }^{\text {REF }}$ & & & 1 \\
\hline Married & 0.355 & 0.232 & $1.427(0.796-2.556)$ \\
\hline Separated/ Divorced & -0.346 & 0.642 & $0.707(0.165-3.042)$ \\
\hline Widowed/ Widower & -0.928 & 0.443 & $0.396(0.037-4.235)$ \\
\hline In a relationship & -0.379 & 0.427 & $0.685(0.269-1.742)$ \\
\hline \multicolumn{4}{|l|}{ Place of residence } \\
\hline North-Central ${ }^{\text {REF }}$ & & & 1 \\
\hline North-East & 0.380 & 0.515 & $1.462(0.467-4.579)$ \\
\hline North-West & 0.229 & 0.507 & $1.257(0.640-2.472)$ \\
\hline South-East & 0.039 & 0.939 & $1.040(0.382-2.835)$ \\
\hline South-South & 0.596 & 0.230 & $1.815(0.685-4.805)$ \\
\hline South-West & 0.538 & 0.055 & $1.713(0.988-2.969)$ \\
\hline \multicolumn{4}{|l|}{ Religion } \\
\hline None ${ }^{\text {REF }}$ & & & 1 \\
\hline Christianity & -1.696 & 0.141 & $0.183(0.019-1.751)$ \\
\hline Islam & -1.001 & 0.397 & $0.368(0.036-3.729)$ \\
\hline Others & 18.658 & 0.999 & \\
\hline \multicolumn{4}{|l|}{ Occupation } \\
\hline Unemployed ${ }^{\text {REF }}$ & & & 1 \\
\hline
\end{tabular}


medRxiv preprint doi: https://doi.org/10.1101/2021.07.30.21261351; this version posted August 1, 2021. The copyright holder for this preprint (which was not certified by peer review) is the author/funder, who has granted medRxiv a license to display the preprint in perpetuity.

It is made available under a CC-BY-ND 4.0 International license.

\begin{tabular}{|c|c|c|c|}
\hline Trader & -0.518 & 0.477 & $0.596(0.143-2.481)$ \\
\hline Teacher & -0.113 & 0.841 & $0.893(0.296-2.692)$ \\
\hline Artisan & -0.371 & 0.636 & $0.690(0.149-3.206)$ \\
\hline Healthcare worker & 0.598 & 0.285 & $1.819(0.608-5.446)$ \\
\hline Information technology & -0.051 & 0.936 & $0.950(0.269-3.358)$ \\
\hline Financial services & -0.263 & 0.679 & $0.769(0.221-2.674)$ \\
\hline Security agent & -0.441 & 0.600 & $0.644(0.124-3.341)$ \\
\hline Agricultural services & 0.178 & 0.807 & $1.195(0.286-4.985)$ \\
\hline Others & 0.284 & 0.572 & $1.328(0.496-3.560)$ \\
\hline \multicolumn{4}{|l|}{ Average income } \\
\hline$<500,000^{\mathrm{REF}}$ & & & 1 \\
\hline $500,000-1$ million & 0.398 & 0.189 & $1.489(0.822-2.700)$ \\
\hline $1-2$ million & 0.528 & 0.108 & $1.696(0.890-3.232)$ \\
\hline $2-3$ million & 0.139 & 0.717 & $1.149(0.541-2.441)$ \\
\hline $3-4$ million & 1.302 & $0.012 *$ & $3.676(1.324-10.212)$ \\
\hline$>4$ million & 0.095 & 0.798 & $1.100(0.530-2.280)$ \\
\hline \multicolumn{4}{|c|}{ Readiness to take vaccine for COVID-19 } \\
\hline Yes & 0.092 & 0.729 & $1.097(0.650-1.851)$ \\
\hline No & -0.088 & 0.782 & $0.916(0.492-1.704)$ \\
\hline Maybe $^{\text {REF }}$ & & & 1 \\
\hline \multicolumn{4}{|c|}{ Vaccines (immunization) are good } \\
\hline Yes & 0.753 & $0.013^{*}$ & $2.122(1.175-3.835)$ \\
\hline No & 0.594 & 0.385 & $1.811(0.474-6.918)$ \\
\hline Maybe $^{\text {REF }}$ & & & 1 \\
\hline \multicolumn{4}{|c|}{ Ever received any vaccine before } \\
\hline Yes & 0.529 & 0.413 & $1.697(0.479-6.016)$ \\
\hline No & -0.179 & 0.808 & $0.836(0.198-3.535)$ \\
\hline Maybe ${ }^{\text {REF }}$ & & & 1 \\
\hline \multicolumn{4}{|l|}{ Knowledge of COVID-19 } \\
\hline Good & 0.947 & $0.019 *$ & $2.578(1.167-5.695)$ \\
\hline Poor ${ }^{\text {REF }}$ & & & 1 \\
\hline
\end{tabular}


medRxiv preprint doi: https://doi.org/10.1101/2021.07.30.21261351; this version posted August 1, 2021. The copyright holder for this preprint (which was not certified by peer review) is the author/funder, who has granted medRxiv a license to display the preprint in perpetuity.

It is made available under a CC-BY-ND 4.0 International license .

Table 7: Predictors of good knowledge of COVID-19

\begin{tabular}{|c|c|c|c|c|c|}
\hline \multirow[t]{2}{*}{ Variable } & \multirow[t]{2}{*}{$\mathbf{B}$} & \multirow[t]{2}{*}{ p value } & \multirow[t]{2}{*}{ OR } & \multicolumn{2}{|c|}{$95 \% \mathrm{CI}$} \\
\hline & & & & Lower & Upper \\
\hline \multicolumn{6}{|l|}{ Age (years) } \\
\hline $18-25$ & 2.011 & $0.008 *$ & 7.474 & 1.697 & 32.920 \\
\hline $26-35$ & 1.105 & 0.052 & 3.019 & 0.990 & 9.204 \\
\hline $36-45$ & 0.852 & 0.101 & 2.345 & 0.847 & 6.494 \\
\hline $46-55$ & 0.819 & 0.193 & 2.267 & 0.661 & 7.781 \\
\hline$>55^{\mathrm{REF}}$ & & & 1 & & \\
\hline \multicolumn{6}{|l|}{ Marital status } \\
\hline Single ${ }^{\text {REF }}$ & & & 1 & & \\
\hline Married & 0.776 & $0.024 *$ & 2.173 & 1.105 & 4.273 \\
\hline Separated/ Divorced & 0.380 & 0.648 & 1.463 & 0.286 & 7.475 \\
\hline Widowed/ Widower & -0.191 & 0.837 & 0.826 & 0.134 & 5.104 \\
\hline In a relationship & 1.846 & 0.076 & 6.331 & 0.825 & 48.615 \\
\hline \multicolumn{6}{|l|}{ Occupation } \\
\hline Unemployed REF $^{\text {Rn }}$ & & & 1 & & \\
\hline Trader & 19.727 & 0.998 & 0.000 & & \\
\hline Teacher & -0.288 & 0.620 & 0.750 & 0.241 & 2.339 \\
\hline Artisan & 0.759 & 0.507 & 2.135 & 0.227 & 20.062 \\
\hline Healthcare worker & 0.904 & 0.105 & 2.471 & 0.829 & 7.365 \\
\hline Information technology & 2.021 & 0.073 & 7.545 & 0.830 & 68.549 \\
\hline Financial services & 0.998 & 0.198 & 2.713 & 0.594 & 12.401 \\
\hline Security agent & -0.264 & 0.778 & 0.768 & 0.123 & 4.800 \\
\hline Agricultural services & 0.021 & 0.979 & 1.021 & 0.211 & 4.948 \\
\hline Others & 0.491 & 0.339 & 1.633 & 0.598 & 4.463 \\
\hline
\end{tabular}

B: Coefficient of Binary logistic regression; OR: Odds ratio; 95\% CI: $95 \%$ Confidence Interval; REF: Reference category; *: p value $<0.05$ 
medRxiv preprint doi: https://doi.org/10.1101/2021.07.30.21261351; this version posted August 1, 2021. The copyright holder for this preprint (which was not certified by peer review) is the author/funder, who has granted medRxiv a license to display the preprint in perpetuity.

It is made available under a CC-BY-ND 4.0 International license .

\section{RESULTS}

A total of 776 participants completed the survey. Most were within the ages of 36-45 years (43.9\%), with $58.1 \%$ males and $40.9 \%$ females. The majority $(53.2 \%)$ had tertiary education with $7.7 \%$ of the respondents being artisans, $8.6 \%$ being teachers and $25 \%$ being health care workers. Most respondents (26.7\%) preferred not to say their annual income with $17.5 \%$ earning less than 500,000 Naira /annum. Zonal representation of respondents (state of origin and place of residence) revealed that most of the respondents were from the southwest zone of the country (47.4\% and 46\% respectively). Most respondents' households were made up of 1-4 persons (48.3\%) while $5.7 \%$ of the respondents had more than 8 persons per household (Table 1).

Overall, majority (90.3\%) of the respondents had a good knowledge of COVID 19 while the remaining 9.7\% had poor knowledge (Figure 1). Most of the respondents $(71.8 \%)$ reported multiple sources of awareness of COVID 19 including the mass media, immediate community, and hospital-related sources. About a quarter of the respondents reported mass media as their only source of awareness (Table 2).

Univariate distribution of the risk perception of respondents revealed that almost all the respondents (98\%) agreed that COVID 19 is a global disease and can affect both adults and children. In addition, most of them (94\%) agreed that as individuals they were doing a lot to protect their family, and majority also agreed that people can die or recover from the disease. Surprisingly, many of the respondents were indifferent towards government and health system response in tackling the disease $(30.4 \%$ and $31.7 \%$ respectively) and majority were also either neutral or disagreed that COVID 19 is a threat to humanity. More than half of the respondents disagreed that the disease affects the elderly with about $30 \%$ indifferent about going to the hospital when ill (Table 3). 
medRxiv preprint doi: https://doi.org/10.1101/2021.07.30.21261351; this version posted August 1, 2021. The copyright holder for this preprint (which was not certified by peer review) is the author/funder, who has granted medRxiv a license to display the preprint in perpetuity. It is made available under a CC-BY-ND 4.0 International license .

Overall, $57 \%$ of the respondents had a positive risk perception of COVID-19 while $43 \%$ had a negative risk perception (Figure 2). Sociodemographic variables such as age, marital status and occupation of the respondents were found to significantly influence the knowledge of the respondents on COVID 19 (Table $4 \mathbf{a}$ and 4b).

Four hundred and eighteen $(59.6 \%)$ of the seven hundred and one respondents with good knowledge about COVID 19 also had positive perception of COVID 19 risk. Contrarily, only twenty-four (32\%) of seventy five subjects with poor COVID 19 knowledge had positive risk perception. Thus, we found a statistically significant relationship between good knowledge and positive risk perception of COVID 19 ( $\mathrm{p}<0.001)$. (Table 5)

Significant predictors of positive risk perception include annual income $(p=0.012, O R=3.676$, CI $1.324-10.212)$, the perception that 'vaccines are good' $(p=0.013, O R=2.122, C I 1.175$ $3.835)$ and good knowledge of COVID $19(p=0.019$, OR $=2.578, C I 1.167-5.695)$. Age, level of education, marital status, place of readiness, religion, occupation, readiness to receive the COVID 19 vaccine and history of previous vaccinations did not significantly predict COVID 19 risk perception. (Table 6)

Variables that significantly predicted good knowledge of COVID 19 were respondents between the ages of 18 to 25 years and married respondents $(p=0.008, O R=7.474, C I 1.697-32.920$ and $p=0.024, O R=2.173, C I 1.105-4.273$ respectively). The occupation of respondents did not significantly predict good knowledge of COVID 19. (Table 7) 
medRxiv preprint doi: https://doi.org/10.1101/2021.07.30.21261351; this version posted August 1, 2021. The copyright holder for this preprint (which was not certified by peer review) is the author/funder, who has granted medRxiv a license to display the preprint in perpetuity.

It is made available under a CC-BY-ND 4.0 International license .

\section{DISCUSSION}

In this study, most of the respondents were between the age group 36-45 years and majority were males. This is similar to findings obtained in related online studies on COVID 19 in Nigeria. ${ }^{26,27}$ This is also reflective of the general demographics of Nigeria which is made up of an expansive population pyramid. ${ }^{28}$ The higher male respondent might also be due to disparity in gender accessibility to internet usage in Nigeria which is currently skewed towards male. ${ }^{29}$ This is particularly so, when you consider a similar study done in south west Nigeria using the traditional methods of sample collection, the study revealed a more male dominated respondent than the female, which is in consonance with the findings of this study. ${ }^{30}$

Three socio demographic variables from the study namely, age, marital status, and occupation had statistically significant association with knowledge of COVID 19. This study revealed that health care workers had good knowledge of COVID 19 compared to other occupations sampled. This is similar to another study in Nigeria that showed that health care workers have good knowledge of COVID $19 .{ }^{31}$ Similar study in the northern Nigeria showed that a quarter of respondents got their information about COVID 19 from health care workers which might also inform the good knowledge of health care workers. ${ }^{32}$ This is not surprising as COVID 19 is a health issue and health care workers have been at the forefront of the management of the disease.

The overall knowledge of our respondents on the outbreak of COVID 19 crisis is high at $90.3 \%$, this is probably due to multiple and modern forms of information dissemination available in Nigeria today. Most of our respondents had information from multiple sources. Available evidence showed that internet penetration seem to be high among young Nigerians and this seems to facilitate health information seeking behaviour. ${ }^{33}$ In this study, social media with other 
medRxiv preprint doi: https://doi.org/10.1101/2021.07.30.21261351; this version posted August 1, 2021. The copyright holder for this preprint (which was not certified by peer review) is the author/funder, who has granted medRxiv a license to display the preprint in perpetuity.

It is made available under a CC-BY-ND 4.0 International license .

electronics media sources were the highest information sources among Nigerians. This was like the findings from other studies done in Nigeria on COVID-19. ${ }^{34,35}$

The knowledge of the respondent in this study was higher than that of those in the study on COVID 19 crisis in sub-Saharan Africa by Adesegun et al that reported 78\%, but comparable to a similar study done on knowledge, attitude, and practice of COVID 19 outbreak, a populationbased study in Iran by Erfani et al with $90 \%$ demonstrating good knowledge among Iranian population surveyed in the study. ${ }^{34,36}$

The younger age among the respondents in Nigeria was a predictor of the good knowledge, which was also noted to be strongly associated with their risk perceptions. In other words, young Nigerians have good knowledge about on-going COVID 19 pandemic and are aware of the risk that this poses to the health in general. These findings were similar to the study findings of Shamir et al which showed that information source was significantly determined by sociodemographic characteristics among young Americans and was also associated with both knowledge and beliefs about the pandemic in the United States. ${ }^{37}$

In this study, the risk perceptions associated with COVID 19 diseases was found to be strongly linked to the knowledge and understanding of the disease as a global burden, which could affect both the young and old, as well as being fatal. The pattern seen in this study has further validated reports from other studies on risk perception on COVID 19 which revealed a significant variability in risk perception seen across the globe. ${ }^{38,39}$ Despite the burden COVID 19 poses globally, more than half of the population in this study are indifferent and do not believe that the government is doing enough to protect the general populace from contracting the virus and this is in consonance with a similar study on COVID $19 .{ }^{40}$ This failure of trust in the government's effort may account for why majority of our respondents don't rely or patronize government's 
medRxiv preprint doi: https://doi.org/10.1101/2021.07.30.21261351; this version posted August 1, 2021. The copyright holder for this preprint (which was not certified by peer review) is the author/funder, who has granted medRxiv a license to display the preprint in perpetuity.

It is made available under a CC-BY-ND 4.0 International license .

health facility as noted in this study, despite government efforts in providing affordable health care to her citizenry. This is also in tandem with a study done in Nigeria which revealed similar findings. ${ }^{40}$

This study also showed that there is a significant association between the amount of knowledge possessed on the disease and the spectrum of the perceived risks regarding COVID 19 across the six geopolitical zones of the country, with good knowledge translating to a higher perception of risks associated with COVID 19 and poor knowledge otherwise. This finding is similar to what was reported in a global study done on global risk perceptions of COVID 19 in 10 countries across the continents of Europe, Asia and America. ${ }^{38}$ Although in Nigeria about a third of the population are of the opinion that government is doing enough to protect them and their family, about the same fraction feel otherwise with the remaining $33 \%$ of the respondents being indifferent to government efforts at ameliorating the effects of COVID 19. This may explain why a significant percentage of the respondents may have adopted for themselves the responsibility of ensuring their safety rather than wait on the government to provide protection for the general populace. These measures being used by individuals may not provide adequate protection against transmission of the disease but rather promote its spread. These are issues which the government agencies task with managing COVID 19 and its complications must investigate. The citizens' response to safety protocols must be consider critically if any major progress is to be seen in curbing the spread of COVID 19, particularly with the paucity of vaccines in Africa and the possibility of the 3rd wave of the disease already being given serious consideration in other parts of the world. ${ }^{41,42}$

This study has identified good knowledge of the disease and immunization against the virus as the two most important predictors of positive risk perception on COVID 19. This may imply that 
medRxiv preprint doi: https://doi.org/10.1101/2021.07.30.21261351; this version posted August 1, 2021. The copyright holder for this preprint (which was not certified by peer review) is the author/funder, who has granted medRxiv a license to display the preprint in perpetuity. It is made available under a CC-BY-ND 4.0 International license .

advocacy on widespread education about the virus and the need for immunization should be embarked upon by the government should any meaningful progress be expected in the fight against the deadly virus.

\section{Limitations}

Findings may be influenced by selection bias because respondents needed access to a smartphone or computer. This may have excluded the poor, elderly who are most vulnerable to COVID-19 this may limit external validity and may have distorted estimation of those willing to take the vaccine.

\section{RECOMMENDATION}

It will be imperative for government and non-governmental organizations to strengthen the capacities of health workers to educate and supply information on COVID 19 to the populace because in this research respondents who are health workers tend to have better knowledge of COVID 19 than respondent who are not.

\section{DECLARATIONS}

\section{Ethics approval}

Ethical approval was obtained from the Health Research Ethics Committee of Federal Medical Center, Gusau, Zamfara State, Nigeria

\section{Consent for publication}

Not applicable 
medRxiv preprint doi: https://doi.org/10.1101/2021.07.30.21261351; this version posted August 1, 2021. The copyright holder for this preprint (which was not certified by peer review) is the author/funder, who has granted medRxiv a license to display the preprint in perpetuity.

It is made available under a CC-BY-ND 4.0 International license.

\section{Availability of data and materials}

The datasets used and/or analyzed during the current study are available from the corresponding author on reasonable request

\section{Competing interests}

The authors declare that they have no competing interests

\section{Funding}

No funding was received for this research

\section{Authors` Contributions}

Conception/design of the study-COO, VKS, OBF, AOO; data collection-UOA, CMI, JCO, OFA, KWO; data analysis and interpretation-EEA, GOP, AOA, OEA; article drafting- BFU, YBA, OFA, AOO; Critical revision of the article- COO, BFU, VKS; final approval of the version to be published-all authors

\section{Acknowledgment}

Oluwafunmike Ruth Olomofe is appreciated for proof-reading the final manuscript. 
medRxiv preprint doi: https://doi.org/10.1101/2021.07.30.21261351; this version posted August 1, 2021. The copyright holder for this preprint (which was not certified by peer review) is the author/funder, who has granted medRxiv a license to display the preprint in perpetuity.

It is made available under a CC-BY-ND 4.0 International license .

\section{REFERENCES}

1. World Health Organization (WHO). Coronavirus Disease (COVID19) Dashboard 2020. https://covid19.who.int/?gclid=EAIaIQobChMIpmCgKqU7gIVumDmCh3R2QH6EAAY ASAAEgLWQfD_BwE Accessed 15 December 2020

2. NCDC Coronavirus COVID-19 Microsite. 2021. https://covid19.ncdc.gov.ng/ Accessed 16 January 2021.

3. COVID-19 Map - Johns Hopkins Coronavirus Resource Center. 2021. https://coronavirus.jhu.edu/map.html Accessed 16 January 2021.

4. Baron S. Galveston; Structure and classification of viruses. Medical microbiology 4th edition.

5. Chen N, Zhou M, Dong X, Qu J, Gong F, Han Y, et al. . "Epidemiological and clinical characteristics of 99 cases of 2019 novel coronavirus pneumonia in Wuhan, China: a descriptive study". Lancet 2020; 395 (10223): 507-513.

6. Huang, C., Wang, Y., Li, X., et al.. Clinical features of patients infected with 2019 novel coronavirus in Wuhan China.The Lancet 2020; 395(10223), 497-506

7. Ilesanmi O, Afolabi A. Perception and practices during the COVID-19 pandemic in an urban community in Nigeria: a cross-sectional study. PeerJ. 2020;8: e10038.

8. Africa Centre for Disease Control and Prevention. Another new coronavirus variant found in Nigeria 2020. [Online]. Available from: https://www.reuters.com/article/ushealth-coronavirus-africa/another-new-coronavirus-variant-found-in-nigeria-says-africacdc-idUSKBN28Y1B7 (Accessed 5 January 2021)

9. Nigerian Centre for Disease Control and Prevention (NCDC). Public Health Advisory Following Increase in Number of COVID-19 Cases in Nigeria. [Online]. Available from: 
medRxiv preprint doi: https://doi.org/10.1101/2021.07.30.21261351; this version posted August 1, 2021. The copyright holder for this preprint (which was not certified by peer review) is the author/funder, who has granted medRxiv a license to display the preprint in perpetuity.

It is made available under a CC-BY-ND 4.0 International license .

https://ncdc.gov.ng/news/280/public-health-advisory-following-increase-in-number-ofcovid-19-cases-in-nigeria (Accessed 10 January 2021)

10. John Hopkins Medicine. Coronavirus and COVID-19: Who is at higher risk? [Online]. Available from: $\quad$ https://www.hopkinsmedicine.org/health/conditions-anddiseases/coronavirus/coronavirus-and-covid19-who-is-at-higher-risk $\quad$ (Accessed 10 January 2021)

11. Ning L, Niu J, Bi X, Yang C, Liu Z, Wu Q, Ning N, Liang L, Liu A, Hao Y, Gao L, and Liu C. The impacts of knowledge, risk perception, emotion and information on citizens' protective behaviors during the outbreak of COVID-19: a cross-sectional study in China. BMC Public Health 2020; 20:1751

12. COVID-19: infection prevention and control guidance (2020). Retrieved from: https://assets.publishing.service.gov.uk/government/uploads/system/uploads/attachment data/file/886668/COVID-19_Infection_prevention_and_control_guidance_complete.pdf

13. Olapegba P, Ayandele O. Survey data of COVID-19-related Knowledge, Risk Perceptions and Precautionary Behavior among Nigerians, Data in Brief (2020), doi: https://doi.org/10.1016/j.dib.2020.105685.

14. Cori L, Bianchi F, Cadum E, Anthonj C.Risk perception and COVID-19. Int. J. Environ. Res. Public Health 2020, 17, 3114

15. Bhagavathula AS., Aldhaleei WA., Rahmani J, Mahabadi MA., Bandari D.K. Novel Coronavirus (COVID-19) Knowledge and Perceptions: A Survey on Healthcare workers. Med Rxiv. 2020;2020.03.09.20033381. 
medRxiv preprint doi: https://doi.org/10.1101/2021.07.30.21261351; this version posted August 1, 2021. The copyright holder for this preprint (which was not certified by peer review) is the author/funder, who has granted medRxiv a license to display the preprint in perpetuity.

It is made available under a CC-BY-ND 4.0 International license .

16. Maiduguri's keke are spreading COVID-19 prevention message (2020). Retrieved from https://reliefweb.int/report/nigeria/maiduguri-s-keke-are-spreading-covid-19-prevention$\underline{\text { messages }}$ [Online Resource]

17. Water Aid Nigeria: Tackling COVID-19 in Nigeria (2020). Retrieved from https://www.wateraid.org/ng/covid-19 [Online Resource]

18. Abdelhafiz A, Mohammed Z, Ibrahim M. Knowledge, Perceptions, and Attitude of Egyptians Towards the Novel Coronavirus Disease (COVID-19) J Community Health. 2020;1(10) https://doi.org/10.1007/s10900-020-00827-7

19. Lagos state threatens to revert to full lock-down (2020). Retrieved from https://nairametrics.com/2020/05/10/lagos-state-threatens-to-revert-to-full-lock-down/ [Online Resource]

20. When a governor believes it's a hoax and ordinary flu (2020). Retrieved from https://gga.org/covid-19-when-a-governor-believes-its-a-hoax-and-ordinary-flu/ [Online Resource]

21. TheGuardian. COVID-19: Why Nigerians fear another lockdown yet defy safety protocols. $\quad$ https://guardian.ng/saturday-magazine/cover/covid-19-why-nigerians-fearanother-lockdown-yet-defy-safety-protocols/ Accessed 14 January 2021

22. Jekel, J., Katz, D., Elmore, J. Sample size, randomization, and probability theory. Epidemiology, Biostatistics and Preventive Medicine. 2001 2nd Ed. Philadelphia: Saunders.

23. National Demographic Health Survey (NDHS). Household population and Housing characteristics National Population Commission (NPC) Federal Republic of Nigeria, Abuja, Nigeria. 2013:11-29 
medRxiv preprint doi: https://doi.org/10.1101/2021.07.30.21261351; this version posted August 1, 2021. The copyright holder for this preprint (which was not certified by peer review) is the author/funder, who has granted medRxiv a license to display the preprint in perpetuity. It is made available under a CC-BY-ND 4.0 International license .

24. Geldsetzer, P. Knowledge and Perceptions of COVID-19 Among the General Public in the United States and the United Kingdom: A Cross-sectional Online Survey. Annals Int Med. 2020. doi: 10.7326/M20-0912

25. Zhong B., Luo W., Li H, Zhang Q., Liu X., Li W., et al. Knowledge, attitudes, and practices towards COVID-19 among Chinese residents during the rapid rise period of the COVID-19 outbreak: a quick online cross-sectional survey. Int $\mathrm{J}$ Biol Sci. 2020;16(10):1745-52.

26. Reuben, R.C., Danladi, M.M.A., Saleh, D.A. et al. Knowledge, Attitudes and Practices towards COVID-19: An Epidemiological Survey in North-Central Nigeria. J Community Health (2020). https://doi.org/10.1007/s10900-020-00881-1

27. Oleribe O, Ezechi O, Osita-Oleribe P, et al Public perception of COVID-19 management and response in Nigeria: a cross-sectional survey $B M J$ Open 2020;10:e041936. doi: 10.1136/bmjopen-2020-041936

28. Nigeria (2021). The world fact book. Available at https://www.cia.gov/the-worldfactbook/countries/nigeria/ (Accessed February, 26 2021)

29. Ibrahim, H. J. (2018). Gender Disparity in Internet Access and Usage in Nigeria: Implication for Development. International Journal of Law, Government and Communication, 3 (12), 41-50.

30. Ilesanmi O, Afolabi A. (2020). Perception and practices during the COVID-19 pandemic in an urban community in Nigeria: a cross-sectional study. PeerJ 8: e10038 DOI $10.7717 /$ peerj. 10038

31. Ejeh, F. E., Saidu, A. S., Owoicho, S., Maurice, N. A., Jauro, S., Madukaji, L., \& Okon, K. O. (2020). Knowledge, attitude, and practice among healthcare workers towards 
medRxiv preprint doi: https://doi.org/10.1101/2021.07.30.21261351; this version posted August 1, 2021. The copyright holder for this preprint (which was not certified by peer review) is the author/funder, who has granted medRxiv a license to display the preprint in perpetuity.

It is made available under a CC-BY-ND 4.0 International license .

COVID-19 outbreak in Nigeria. Heliyon, e05557. doi:10.1016/j.heliyon.2020.e05557

32. Habib M. A, Dayyab F. M, Iliyasu G, Habib AG (2021) Knowledge, attitude and practice survey of COVID-19 pandemic in Northern Nigeria. PLoS ONE 16(1): e0245176. doi:10.1371/journal.pone.0245176

33. Obasola O. I, Agunbiade O. M, Online Health Information Seeking Pattern among Undergraduates in a Nigerian University. SAGE Open. January 2016. doi:10.1177/2158244016635255

34. Adesegun, O. A., Binuyo, T., Adeyemi, O., Ehioghae, O., Rabor, D. F., Amusan, O., Akinboboye, O., Duke, O. F., Olafimihan, A. G., Ajose, O., Idowu, A. O., \& Abiodun, O. (2020). The COVID-19 Crisis in Sub-Saharan Africa: Knowledge, Attitudes, and Practices of the Nigerian Public. The American Journal of Tropical Medicine and Hygiene, 103(5), 1997-2004. Retrieved Feb 25, 2021, from https://www.ajtmh.org/view/journals/tpmd/103/5/article-p1997.xml

35. Hager E, Odetokun IA, Bolarinwa O, Zainab A, Okechukwu O, Al-Mustapha AI. Knowledge, attitude, and perceptions towards the 2019 Coronavirus Pandemic: A binational survey in Africa. PLoS One. 2020 Jul 29; 15(7):e0236918. doi: 10.1371/journal.pone.0236918.

36. Erfani A, Shahriarirad R, Ranjbar K, Mirahmadizadeh A \& Moghadami M. Knowledge, Attitude and Practice toward the Novel Coronavirus (COVID-19) Outbreak: A Population-Based Survey in Iran. [Preprint]. Bull World Health Organ. E-pub: 30 March 2020. doi: http://dx.doi.org/10.2471/BLT.20.256651 
medRxiv preprint doi: https://doi.org/10.1101/2021.07.30.21261351; this version posted August 1, 2021. The copyright holder for this preprint (which was not certified by peer review) is the author/funder, who has granted medRxiv a license to display the preprint in perpetuity. It is made available under a CC-BY-ND 4.0 International license .

37. Ali S. H, Foreman J, Tozan Y, Capasso A, Jones A. M, Di-Clemente R. J. Trends and Predictors of COVID-19 Information Sources and Their Relationship With Knowledge and Beliefs Related to the Pandemic: Nationwide Cross-Sectional Study. JMIR Public Health Surveillance 2020;6(4):e21071

38. Sarah Dryhurst et al (2020) Risk perceptions of COVID-19 around the world, Journal of Risk Research, 23:7-8, 994-1006, DOI: 10.1080/13669877.2020.1758193

39. Rohde R., (2020). Novel Coronavirus (2019-nCoV) update: Uncoating the Virus. America Society of Microbiologist.

40. Olomofe C, Shoyemi K, Omoworare B, Adeyinka O, Ajumuka E., et.al (2021). Predictors of Uptake of a Potential Covid 19 Vaccine among Nigeria Adults, Journal of Vaccine and Vaccination. Vol. 12 Issue. 1 No: 1000442

41. Xolisa Phillip. Covid 19 vaccines in Africa: MO Ibrahim and others Lament sorry state of access; The Africa repot; Jan, 2021

42. Baker H. A, Safavynia S. A, Evered L. A. (2020) British Journal on Anaesthesia; S00070912(20)30849. Available at Pubmed.ncbi.nlm.nih.gov (Accessed February, 26 2021) 